\title{
A longitudinal study on determinants of HPV vaccination uptake in parents/ guardians from different ethnic backgrounds in Amsterdam, the Netherlands
}

Catharina J. Alberts ${ }^{1,2^{*}}$, Maarten F. Schim van der Loeff ${ }^{1,2}$, Yvonne Hazeveld $^{3}$, Hester E. de Melker ${ }^{4}$, Marcel F. van der Wal ${ }^{5}$, Astrid Nielen ${ }^{3}$, Fatima El Fakiri ${ }^{5}$, Maria Prins ${ }^{1,2}$ and Theo G. W. M. Paulussen ${ }^{6}$

\begin{abstract}
Background: Human papillomavirus (HPV) vaccination coverage in the Netherlands is low ( 60\%) compared to other childhood vaccinations (>90\%), and even lower among ethnic minorities. The aim of this study was to explore the possible impact of ethnicity on the determinants of both HPV vaccination intention and HPV vaccination uptake among parents/guardians having a daughter that is invited for the HPV vaccination.
\end{abstract}

Methods: In February 2014, parents/guardians living in Amsterdam were invited to complete a questionnaire about social-psychological determinants of their decision making process regarding the HPV vaccination of their daughter and socio-demographic characteristics. This questionnaire was sent approximately one month before the daughter was scheduled to receive her first HPV vaccine dose. Their daughters' HPV vaccination status was retrieved from the national vaccination database. We distinguished four ethnic groups: Dutch (NL), Surinamese, Netherlands Antillean, and Aruban (SNA), Middle-Eastern and North-African (MENA), and Other. To assess the impact of determinants on both intention and uptake, linear and logistic regression analyses were used respectively. Missing data were imputed using multiple imputation by chained equation.

Results: In total 1,309 parents/guardians participated (33\% participation rate). In all groups we found the mothers' intention to be the strongest predictor of their daughters' HPV vaccination uptake. Explained variance of uptake was highest in the NL-group (pseudo- $R^{2}: 0.56$ ) and lower in the other ethnic groups (pseudo- $R^{2}$ varied between 0.23 and 0.29). The lower explained variance can be attributed to the relative large proportion of participants with a positive intention that finally did not go for vaccination in the SNA-group (11\%) and MENA-group (30\%). Explained variance $\left(R^{2}\right)$ of intention varied between 0.66 and 0.77 across ethnic groups, and was best explained by the proximal social-psychological determinants. The strength of association of these determinants with both intention and uptake were largely similar across ethnic groups.

(Continued on next page)

\footnotetext{
*Correspondence: nalberts@ggd.amsterdam.nl

'Department of Infectious Diseases, Research and Prevention, Public Health

Service (GGD) of Amsterdam, Nieuwe Achtergracht 100, 1018, WT,

Amsterdam, The Netherlands

${ }^{2}$ Department of Internal Medicine, Division of Infectious Diseases, Center for

Infection and Immunity Amsterdam (CINIMA), Academic Medical Center

(AMC), Amsterdam, The Netherlands

Full list of author information is available at the end of the article
} 
(Continued from previous page)

Conclusion: We conclude that the same determinants should be targeted in the different ethnic groups, although the mode of delivery of the intervention needs to be tailored to the different cultural backgrounds. Further research is needed to explain the observed discrepancy between intention and uptake, especially among parents/guardians in the non-Dutch groups.

Keywords: HPV, Vaccination acceptability, Vaccination uptake, Population-based, Ethnicity, the Netherlands

\section{Background}

The national immunization program (NIP) of the Netherlands is a voluntary and free-of-charge program with an overall high childhood vaccination uptake that exceeds 90\% [1]. However, in 2009 when the HPV vaccination was added to the NIP, the uptake of this particular vaccination was drastically lower: $52 \%$ in 2009 [2], increasing to $61 \%$ in 2014 [1]. Lower uptake has been observed in certain ethnic minorities; e.g. in 2009 an uptake of $44 \%, 38 \%$, and $24 \%$ was observed among girls of which both parents were born in Surinam, Turkey, or Morocco, respectively [3]. This is especially worrying as these groups are known to have a higher incidence of cervical cancer compared to the native Dutch population [4]. Similar ethnic disparities are observed around the globe [5-9].

In previous studies investigating HPV vaccination acceptability in the Netherlands, common predictors of HPV vaccination intention appeared to be attitude, concerns about vaccination effectiveness and safety, feelings of ambivalence towards HPV vaccination, anticipated regret, and normative beliefs $[7,10,11]$. Factors that did not appear to have an effect on HPV vaccination acceptability were knowledge and knowing someone with an abnormal Pap test $[10,12-14]$. Studies focussing on HPV vaccination acceptability among ethnic minorities are mostly small and/or struggle with answering why differences in uptake between ethnicities exist $[9,11,15,16]$. The aim of this study is to explore the process of decision making by parents/guardians from different ethnic backgrounds about the HPV vaccination uptake of their daughter. The following research questions were central in this study: (1) What are the determinants in the process of parental decision making about the HPV vaccination of their daughter?, and (2) Do these determinants differ between parents/guardians from different ethnic backgrounds?

\section{Methods}

In the Netherlands the bivalent HPV vaccine (Cervarix) is offered since 2009 by the National Immunization Programme (NIP). In 2014, the vaccination schedule was reduced from three to two doses in line with guidelines of the European Medicine Agency. All girls are invited for HPV vaccination in the year they turn 13 years old.
Girls are invited to come to a large public venue designated by the Public Health Service (e.g. sports centers and child-and parent centers). During this vaccination moment no personal consultation is offered and no consent of the parent is necessary to obtain the HPV vaccination.

\section{Participants}

All parent/guardians with a daughter born in 2001 living in the district of the Youth Health Service of the Public Health Service of Amsterdam were invited for this study (2014). They were selected from a database of the Youth Health Service of the Public Health Service of Amsterdam which contains the most up to date information of all girls residing in Amsterdam. The selection of the girls eligible for this study was done one month before they received the invitation of the HPV vaccination. Parents/guardians were able to participate only once. A computer tablet was raffled among participants. The study was approved by the Ethics Committee of the Academic Medical Centre (W2013_257).

\section{Invitation procedure}

Parents/guardians were invited to complete a questionnaire approximately one month before their daughter was scheduled to receive her first HPV vaccine dose (see Additional file 1: Figure S1 for key dates of the recruitment period). Participants provided informed consent for this study by completing and returning the questionnaire. Since our primary aim was to predict the outcome of their decision making regarding the HPV vaccination of their daughter, we restricted our analyses to parents/ guardians who returned the questionnaire before their scheduled vaccination day (see Additional file 1: Figure S2 for a flow diagram).

The questionnaire could be completed online or on paper and was available in three languages Dutch, Turkish, and English. All parents/guardians received a Dutch version of the questionnaire, and based on the country of birth of one of the parents they also received an English or a Turkish version.

In order to increase the response rate among our target population we formed a team of research assistants fluent in Dutch and at least one other relevant language (i.e. Turkish, Berber, Arabic, English, Spanish, or Twi (a 
local language in Ghana)). Based on the country of birth of the parents/guardians, the research assistants were matched to the language presumably spoken by the parents/guardians. The assistants attended a comprehensive training in which they were instructed about: (i) the general background of the HPV vaccination, (ii) what information they were allowed to provide to the parents/guardians in order to not influence parents'/ guardians' responses to the questionnaire, (iii) the content of the questionnaire, and (iv) relevant other logistic aspects concerning data collection and the HPV vaccination of their daughter. The assistants called parents/guardians to explain the rationale behind the study, to offer assistance with completing the questionnaire if necessary, and/or to remind them in case of an initial non-response. If parents asked information regarding the HPV vaccination, the research assistants only gave the information that was provided in the invitation letter for the HPV vaccination and the information leaflet of this study (in practice, most questions concerned location and time of vaccination and concerns regarding the safety of the HPV vaccine).

\section{Questionnaire}

The questionnaire was based on a questionnaire initially developed by van Keulen et al. [10] which was successfully applied in a study on HPV vaccination intention among parents/guardians with a native Dutch background. Adaptations were based on one-on-one interviews and focus groups with native Dutch, Moroccan, Turkish, Ghanaian, and Surinamese parents, separately. The Reasoned Action approach [17], Social Cognitive Theory [18], and the Health Belief Model [19] constituted the theoretical framework of this study. The adapted questionnaire was pretested among parents/ guardians and subsequently revised.

\section{Ethnic group}

The ethnic background of participants was based on country of birth of both parents as described previously [20]. In short, a girl was classified as non-Dutch if at least one parent was born outside the Netherlands. If the mother and the father did not have the same country of birth, the country of birth of the mother was decisive. Based on their ethnicity an individual was categorized into one of the following ethnic groups; (i) Dutch (NL), (ii) Surinamese, Netherlands Antillean, and Aruban (SNA), (iii) Middle Eastern and North African (MENA) (including Turkish), and (iv) other. The MENA was grouped by the region grouping of the WHO (except for Turkey) [21] and was mainly composed of individuals with a Moroccan $(n=109)$, Turkish $(n=67)$, and Egyptian $(n=21)$ ethnicity. The category Other consisted mainly of
Indonesians $(n=35)$, Ghanaians $(n=32)$, and Germans $(n=15)$.

\section{HPV and childhood vaccination intention and uptake} Consent to retrieve the vaccination status of the daughter was obtained by posing a separate question asking whether the parent/guardian agreed if we would retrieve the HPV and childhood vaccination status of their daughter from Praeventis. Praeventis is a national database for monitoring childhood vaccinations in the Netherlands [22]. HPV vaccination status was dichotomized: (i) Received one or both HPV vaccinations, versus (ii) Received no HPV vaccination. Intention was assessed by a composite score of two 5-point Likert-type items (Cronbach $\alpha=0.96$ ).

\section{Determinants of HPV vaccination intention and uptake}

According to their theoretically expected cause-effect relation, socio-psychological determinants of HPV vaccination intention and HPV vaccination uptake were subdivided into proximal determinants versus distal determinants.

Proximal determinants are theoretically expected to impact the parents'/guardians' intention most directly. These were: general attitude, vaccination-related beliefs, negative outcome expectancies, risk perception (e.g. perceived susceptibility when not vaccinated against HPV), anticipated regret, perceived relative effectiveness of the HPV vaccination, subjective norms, descriptive norm, and self-efficacy.

The impact of distal determinants on the parents'/ guardians' intention are expected to be indirect. These were: knowledge, confidence in authorities, ambivalence about the HPV vaccination, habit strength, information processing, amount of information processed, subjective evaluation of the received information about the HPV vaccination, past experience with cervical cancer (self or close relative), and sociodemographic characteristics. Socio-demographics included age, gender, educational level, religion (no religion, Protestantism, Catholicism, Islam, and other), and ethnicity. For analyses religion was dichotomised into religious versus non-religious.

See Additional file 1: Table S1 for more details about the items used per construct, response format, and the internal consistency of composite variables. In short, all socio-psychological determinants were assessed by a composite score (except for risk perception and anticipated regret). Composite scores were calculated when the items showed sufficient internal consistency (Chronbach's $\alpha>0.60$ ). The scores were computed by summing all the items of the construct and dividing the sum by the number of items. Scores were rescaled from -2 to 2 , where appropriate. 


\section{Statistical methods}

Response rate was explored by country of birth of the mother of the girl invited for the HPV vaccination; the country of birth of the mother was grouped into the same four groups as used for ethnicity of the participant. We compared HPV vaccination status, HPV vaccination intention, past childhood vaccination status, and sociodemographics by ethnicity. Descriptive analyses were only computed for complete cases.

Bivariable and multivariable analyses were based on multiple imputed datasets. We used multiple imputation to account for missing data [23]. The percentage of missing values per variable varied between $<1$ and $7 \%$, except for subjective norm, for which $33 \%$ of participants did not answer at least one of the individual items. Most unanswered questions were presumably not answered because of the length of the questionnaire, and participants seemed to skip questions that appeared similar to them. We observed that participants with a lower intention to vaccinate and/or with a non-Dutch ethnicity had more data missing, compared to participants with a high intention and/or Dutch ethnicity. We used multiple imputation to create 15 multiple imputed datasets. Model parameters were combined using Rubin's rule, combined estimates are presented in the tables [24].

HPV vaccination uptake and HPV vaccination intention were both used as criterion variable (i.e. outcome variable). Determinants of these two criterion variables, HPV vaccination intention and $\mathrm{HPV}$ vaccination uptake, were investigated using linear and logistic regression analyses, respectively. The multivariable analyses with uptake as the criterion variable were executed in three steps, by consecutively adding the following three sets of determinants: intention, proximal determinants, and distal determinants. Determinants were only included if the bivariable association with the criterion variable was $p<0.25$. To obtain a parsimonious model, backward selection was executed during each step in which a new set of determinants was offered to the model $(p<0.05)$. Multivariable analyses with $\mathrm{HPV}$ vaccination intention as the criterion variable were executed according to the same theory-based order. In both final multivariable models we tested for interaction effects between ethnic group and each determinant $(p<0.05)$. Statistical analyses were performed in Stata 14 [25].

\section{Results}

\section{Response rate}

Parents/guardians of 4,216 girls were invited to participate in this study. The response rate was highest among mothers born in the Netherlands (37\%), followed by the Other group (34\%), SNA group (31\%) and with the lowest response rate among mothers born in one of the
Middle East or North African countries (20\%) (see Additional file 1: Table S2 for more detail).

Of all parents/guardians invited, 1,362 (33\%) returned a questionnaire of whom 1,317 (96\%) returned the questionnaire before the first scheduled HPV vaccination day. Ethnicity was missing for 8 participating parents/ guardians. This resulted in a net-response of 1,309 participants (see Additional file 1: Figure S2 for details). HPV vaccination uptake among these 1,309 participants was higher when compared to the overall uptake in Amsterdam in 2014 (76\% in complete case analyses, 73\% in the imputed dataset, and $51 \%$ in the total invited population, see Additional file 1: Figure S3).

\section{Baseline characteristics and differences between ethnic groups in complete cases}

The majority of participants were mothers/female guardians $(88 \%)$, median age was 45 years (inter quartile range (IQR): $42-48$ ), and $28 \%$ received higher education. On average, the NL-group was oldest (median 46 (IQR: 4349)) and reported the highest level of education (34\%), $74 \%$ of the NL-group reported not to be religious, while in the MENA-group $80 \%$ reported to be Islamic, and, finally in the NL-group only $7 \%$ received help when completing the questionnaire while this was $43 \%$ in the MENA-group (see Table 1).

Overall, the mean HPV vaccination intention was high (1.20, standard deviation, SD 1.17) but differed across ethnic groups $(p<0.001)$, with the lowest intention found in the MENA-group (mean 0.68; SD: 1.30). In the NL-group we found the largest fraction of daughters receiving two HPV vaccine doses (84\%), while the SNAgroup had the largest fraction of daughters that received only one dose (13\%) and the MENA-group had the largest fraction of non-vaccinated daughters (41\%). Overall past childhood vaccination uptake was 95\% (Table 1).

\section{Determinants of HPV vaccination uptake by ethnic group}

In the NL-group we found in multivariable analyses the following determinants to be significantly associated with HPV vaccination uptake (Table 2): intention $(\mathrm{OR}=5.67 ; 95 \% \mathrm{CI}=4.10,7.85)$ and past childhood vaccination uptake $(\mathrm{OR}=10.43 ; 95 \% \mathrm{CI}=3.05,35.69)$. In addition to the determinants found in the NLgroup, we found habit strength $(\mathrm{OR}=2.32 ; 95 \% \mathrm{CI}=$ $1.13,4.78)$ to be significantly associated in the SNAgroup. In the Other-group subjective norms $(\mathrm{OR}=3.52$; $95 \% \mathrm{CI}=1.28,9.67)$ and information processing ( $\mathrm{OR}=$ $0.43 ; 95 \% \mathrm{CI}=0.22,0.83$ ) were significantly associated. No additional determinants were found in the MENA-group. See Additional file 1: Table S3 for the selection of the variables used as input for the multivariable analyses.

The explained fraction (pseudo- $\mathrm{R}^{2}$ ) of HPV vaccination was 0.56 in the NL-group $(0.54$ by intention 
Table 1 Baseline socio-demographic characteristics, HPV vaccination intention, actual childhood vaccination and HPV vaccination uptake, by ethnic group of the parents/guardians, HPV vaccination acceptability study in Amsterdam, the Netherlands, 2014

\begin{tabular}{|c|c|c|c|c|c|c|c|c|c|c|c|c|c|c|}
\hline \multicolumn{15}{|l|}{ Region of origin } \\
\hline \multirow[b]{3}{*}{ Socio-demographic characteristics } & \multirow{2}{*}{\multicolumn{2}{|c|}{$\begin{array}{l}\mathrm{NL} \\
(n=723)\end{array}$}} & \multirow{2}{*}{\multicolumn{2}{|c|}{$\begin{array}{l}\text { SNA } \\
(n=126)\end{array}$}} & \multirow[b]{3}{*}{$p \vee s N$} & \multirow{2}{*}{\multicolumn{2}{|c|}{$\begin{array}{l}\text { MENA } \\
(n=237)\end{array}$}} & \multirow[b]{3}{*}{$p$ vs NL } & \multirow{2}{*}{\multicolumn{2}{|c|}{$\begin{array}{l}\text { Other } \\
(n=223)\end{array}$}} & \multirow[b]{3}{*}{$p$ vs NL } & \multirow{2}{*}{\multicolumn{2}{|c|}{$\begin{array}{l}\text { Total } \\
(n=1309)\end{array}$}} & \multirow[b]{3}{*}{ p overall } \\
\hline & & & & & & & & & & & & & & \\
\hline & $\mathrm{n}$ & $\%$ & $\mathrm{n}$ & $\%$ & & $\mathrm{n}$ & $\%$ & & $\mathrm{n}$ & $\%$ & & $\mathrm{n}$ & $\%$ & \\
\hline \multicolumn{15}{|l|}{ Gender } \\
\hline Female & 630 & $90 \%$ & 108 & $89 \%$ & 0.905 & 180 & $82 \%$ & 0.002 & 190 & $89 \%$ & 0.863 & 1108 & $88 \%$ & 0.017 \\
\hline Male & 73 & $10 \%$ & 13 & $11 \%$ & & 40 & $18 \%$ & & 23 & $11 \%$ & & 149 & $12 \%$ & \\
\hline \multicolumn{15}{|l|}{ Age } \\
\hline Median (IQR) & 46 & $(43-49)$ & 43 & $(39-47)$ & $<0.001$ & 42 & $(37-47)$ & $<0.001$ & 45 & $(41-48)$ & $<0.001$ & 45 & $(42-48)$ & $<0.001$ \\
\hline$\leq 43$ year & 203 & $29 \%$ & 61 & $51 \%$ & $<0.001$ & 119 & $58 \%$ & $<0.001$ & 86 & $42 \%$ & 0.004 & 469 & $38 \%$ & $<0.001$ \\
\hline 44-47 year & 253 & $36 \%$ & 32 & $27 \%$ & & 41 & $20 \%$ & & 63 & $30 \%$ & & 389 & $32 \%$ & \\
\hline$\geq 48$ year & 238 & $34 \%$ & 27 & $23 \%$ & & 45 & $22 \%$ & & 58 & $28 \%$ & & 368 & $30 \%$ & \\
\hline \multicolumn{15}{|l|}{ Education $^{a}$} \\
\hline Low & 204 & $29 \%$ & 71 & $60 \%$ & $<0.001$ & 137 & $65 \%$ & $<0.001$ & 80 & $38 \%$ & 0.033 & 492 & $40 \%$ & $<0.001$ \\
\hline Intermediate & 255 & $37 \%$ & 34 & $29 \%$ & & 43 & $20 \%$ & & 61 & $29 \%$ & & 393 & $32 \%$ & \\
\hline High & 236 & $34 \%$ & 13 & $11 \%$ & & 32 & $15 \%$ & & 67 & $32 \%$ & & 348 & $28 \%$ & \\
\hline \multicolumn{15}{|l|}{ Religion } \\
\hline No religion & 515 & $74 \%$ & 19 & $16 \%$ & $<0.001$ & 11 & $5 \%$ & $<0.001$ & 94 & $46 \%$ & $<0.001$ & 639 & $52 \%$ & $<0.001$ \\
\hline Protestantism $^{\mathrm{b}}$ & 105 & $15 \%$ & 50 & $42 \%$ & & 13 & $6 \%$ & & 41 & $20 \%$ & & 209 & $17 \%$ & \\
\hline Catholicism $^{c}$ & 67 & $10 \%$ & 17 & $14 \%$ & & 19 & $9 \%$ & & 40 & $20 \%$ & & 143 & $12 \%$ & \\
\hline Islam & 2 & $0 \%$ & 16 & $13 \%$ & & 168 & $80 \%$ & & 13 & $6 \%$ & & 199 & $16 \%$ & \\
\hline Other & 6 & $1 \%$ & 17 & $14 \%$ & & 0 & $0 \%$ & & 16 & $8 \%$ & & 39 & $3 \%$ & \\
\hline \multicolumn{15}{|c|}{ Language used to complete the questionnaire } \\
\hline Dutch & 723 & $100 \%$ & 126 & $100 \%$ & N.A. & 194 & $82 \%$ & $<0.001$ & 199 & $89 \%$ & $<0.001$ & 1242 & $95 \%$ & $<0.001$ \\
\hline Another language than Dutch ${ }^{d}$ & 0 & $0 \%$ & 0 & $0 \%$ & & 43 & $18 \%$ & & 24 & $11 \%$ & & 67 & $5 \%$ & \\
\hline \multicolumn{15}{|c|}{ Received help to complete the questionnaire } \\
\hline Did not receive help & 649 & $93 \%$ & 104 & $86 \%$ & 0.011 & 124 & $57 \%$ & $<0.001$ & 160 & $75 \%$ & $<0.001$ & 1037 & $83 \%$ & $<0.001$ \\
\hline Received help ${ }^{e}$ & 50 & $7 \%$ & 17 & $14 \%$ & & 94 & $43 \%$ & & 54 & $25 \%$ & & 215 & $17 \%$ & \\
\hline \multicolumn{15}{|l|}{ Way of completing the questionnaire } \\
\hline On paper & 401 & $55 \%$ & 70 & $56 \%$ & 0.985 & 158 & $67 \%$ & 0.002 & 137 & $61 \%$ & 0.115 & 766 & $59 \%$ & 0.015 \\
\hline Online & 322 & $45 \%$ & 56 & $44 \%$ & & 79 & $33 \%$ & & 86 & $39 \%$ & & 543 & $41 \%$ & \\
\hline \multicolumn{15}{|l|}{ Intention (5 point-scale, range -2 to 2 ) } \\
\hline \multirow[t]{2}{*}{ Mean (SD) } & 1.30 & 1.17 & 1.33 & 0.92 & 0.850 & 0.68 & 1.30 & $<0.001$ & 1.31 & 0.97 & 0.903 & 1.20 & 1.17 & $<0.001$ \\
\hline & $n$ & $\%$ & $\mathrm{n}$ & $\%$ & & $\mathrm{n}$ & $\%$ & & $n$ & $\%$ & & $n$ & $\%$ & \\
\hline \multicolumn{15}{|l|}{ HPV vaccination status } \\
\hline No vaccination & 84 & $13 \%$ & 16 & $16 \%$ & $<0.001$ & 72 & $41 \%$ & $<0.001$ & 33 & $17 \%$ & 0.031 & 205 & $18 \%$ & $<0.001$ \\
\hline One vaccination & 22 & $3 \%$ & 13 & $13 \%$ & & 13 & $7 \%$ & & 13 & $7 \%$ & & 61 & $5 \%$ & \\
\hline Two vaccinations & 539 & $84 \%$ & 71 & $71 \%$ & & 92 & $52 \%$ & & 146 & $76 \%$ & & 848 & $76 \%$ & \\
\hline Infant, toddler and preschool child & ren va & accination & & & & & & & & & & & & \\
\hline Not all & 13 & $2 \%$ & 7 & $7 \%$ & 0.004 & 5 & $3 \%$ & 0.515 & 17 & $9 \%$ & $<0.001$ & 42 & $4 \%$ & $<0.001$ \\
\hline All & 632 & $98 \%$ & 93 & $93 \%$ & & 172 & $97 \%$ & & 175 & $91 \%$ & & 1072 & $96 \%$ & \\
\hline
\end{tabular}


Table 1 Baseline socio-demographic characteristics, HPV vaccination intention, actual childhood vaccination and HPV vaccination uptake, by ethnic group of the parents/guardians, HPV vaccination acceptability study in Amsterdam, the Netherlands, 2014 (Continued)

\begin{tabular}{|c|c|c|c|c|c|c|c|c|c|c|c|c|c|c|}
\hline \multicolumn{15}{|c|}{ School-age vaccination ${ }^{9}$} \\
\hline Not all & 24 & $4 \%$ & 5 & $5 \%$ & 0.538 & 6 & $3 \%$ & 0.835 & 14 & $7 \%$ & 0.031 & 49 & $4 \%$ & 0.169 \\
\hline All & 621 & $96 \%$ & 95 & $95 \%$ & & 171 & $97 \%$ & & 178 & $93 \%$ & & 1065 & $96 \%$ & \\
\hline \multicolumn{15}{|c|}{ Overall childhood vaccination status ${ }^{\mathrm{h}}$} \\
\hline Not all & 24 & $4 \%$ & 8 & $8 \%$ & 0.050 & 8 & $5 \%$ & 0.626 & 20 & $10 \%$ & $<0.001$ & 60 & $5 \%$ & 0.002 \\
\hline All & 621 & $96 \%$ & 92 & $92 \%$ & & 169 & $95 \%$ & & 172 & $90 \%$ & & 1054 & $95 \%$ & \\
\hline
\end{tabular}

* $p$-values of categorical variables are based on Pearson's chi-square test for categorical variables, $p$-values for continuous variables when comparing one of the "region of origin" categories with the Dutch based on the $t$-test $p$-values for continuous variables overall are based on the Kruskall-Wallis test. For calculation of the $p$-values missing categories were excluded

${ }^{a}$ Education was based on the highest level of education and categorized into low (no education, primary school), intermediate (lower general secondary school, higher general secondary school, or secondary vocational school) and high (university-preparatory school, polytechnic, or university)

b Includes all Christian religions not belonging to the Catholic category

Includes Coptic Christian and Greek- orthodox

${ }^{\mathrm{d}}$ Other languages used were Turkish $(n=20)$, English $(n=43)$, Arabic $(n=3)$ or Twi $(n=1)$. Written questionnaire was available in Dutch, English and Turkish eparticipants indicated to have received help to complete the questionnaire, i.e. partner $(n=48)$, daughter/son ( $n=144)$ or different $(n=23)$

${ }^{f} G i r l$ is vaccinated for all vaccinations given between 0 and 4 years of age (without exceptions), which are DTaP: diphtheria-tetanus-acellular pertussis vaccine; HiB: Haemophilus influenzae type b vaccine; MMR: measles-mumps-rubella vaccine (NB; PCV: pneumococcal conjugate vaccine - > was introduced in 2006 and therefore not applicable for this cohort.)

${ }^{\mathbf{g}}$ Girl is vaccinated for all vaccinations given at 9 years of age (without exceptions), which are DTP: diphtheria-tetanus vaccine; MMR: measles-mumps-rubella vaccine

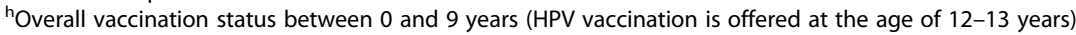

Data are missing for gender $(n=52)$, age $(n=83)$, education $(n=76)$, religion $(n=80)$, received help $(n=57)$ and intention ( $n=20)$. Data are missing for childhood and HPV vaccination status of those girls whose parents/guardians did not consent to obtain their vaccination status from Praeventis, the national database for monitoring the childhood vaccinations in the Netherlands)

Abbreviations: IQR interquartile range, DPTP diphtheria, pertussis, tetanus and poliomyelitis, MMR measles, mumps and rubella, HPV human papillomavirus, SD standard deviation. NL denotes participants with a Dutch ethnicity, SNA denotes participants with a Surinamese, Netherlands Antillean or Aruban ethnicity, MENA denotes participants with a Middle Eastern or North African ethnicity (including Turkish participants), Other denotes participants from all other ethnicities. $p$-values/significance levels are indicated in the columns " $p$ vs NL" and "p overall". The values thatare significant at a level $p<0.05$ are now indicated in bold

and an additional 0.02 by distal determinants); 0.28 in the SNA-group ( 0.20 by intention and an additional 0.08 by distal determinants); 0.23 in the MENA-group (all by intention); and 0.29 in the Other-group $(0.22$ by intention and an additional 0.03 by proximal determinants, and an additional 0.04 by distal determinants).

\section{Differences between determinants of HPV vaccination uptake by ethnic group}

We tested whether the magnitude of the association of determinants of HPV vaccination uptake differed significantly in the non-Dutch groups compared to the NLgroup (Table 3). The association of intention with uptake was significantly lower in the MENA-group $(p=0.020)$ and Other-group $(p=0.042)$ when compared to the NLgroup; the association of subjective norms with uptake was significantly lower in the SNA-group $(p=0.025)$ and MENA-group $(p=0.002)$ when compared to the NL-group; habit strength was significantly lower in the MENA-group when compared to the NL-group $(p<0.001)$.

\section{Relation between intention and uptake}

Among the participants with a positive intention to vaccinate against $\mathrm{HPV}$ we observed that $4 \%$ in the NL-group, $11 \%$ in the SNA-Group (when compared to NL-group $p=0.002), 30 \%$ in the MENA-group (MENA- vs NL-group $p<0.001$ ), and $11 \%$ in the Other-group (Other- vs NL-group $p<0.001$ ) ultimately did not go for the HPV vaccination (inclined abstainers). Among the participants with a negative intention we observed that that $11 \%$ in the NL-group, $7 \%$ in the SNA-Group (SNA- vs NL-group $p=0.606$ ), $6 \%$ in the MENA-group (MENA- vs NL-group $p=$ 0.621 ) and $19 \%$ in the Other-group (Other- vs NLgroup $p=0.298$ ) did go for the HPV vaccination (disinclined actors).

\section{Determinants of HPV vaccination intention by ethnic group}

In multivariable analyses we found the following determinants to be significantly associated with HPV vaccination intention in the NL-group (Table 4): (1) attitude $(B=0.66 ; 95 \% \quad C I=0.57,0.75), \quad(2)$ beliefs $(B=0.36$; $95 \% \mathrm{CI}=0.24,0.49)$, (3) subjective norms $(B=0.49$; 95\% $C I=0.35,0.63),(4)$ ambivalence $(B=-0.08 ; 95 \%$ $\mathrm{CI}=-0.13,-0.03),(5)$ information processing $(B=-0.10$; $95 \% \mathrm{CI}=-0.15,-0.04)$, (6) subjective evaluation of the information ( $\beta=0.23$; $95 \% \mathrm{CI}=0.12,0.34$ ), (7) having an older daughter for whom they already had taken a decision about the HPV vaccination (the same decision was taken for the younger daughter) $(\beta=0.92 ; 95 \% \mathrm{CI}=$ $0.65,1.19)$, (8) education (intermediate education $[\beta=0.14$; 
Table 2 HPV vaccination uptake: multivariable logistic regression analyses of social-psychological, socio-demographic and other factors. HPV vaccination acceptability study among parents/guardians, in Amsterdam, the Netherlands, 2014

\begin{tabular}{|c|c|c|c|c|c|c|c|c|}
\hline & \multicolumn{8}{|c|}{ Multivariable $e^{a}$} \\
\hline & \multicolumn{2}{|l|}{$\begin{array}{l}\mathrm{NL} \\
(n=723)\end{array}$} & \multicolumn{2}{|l|}{$\begin{array}{l}\text { SNA } \\
(n=126)\end{array}$} & \multicolumn{2}{|l|}{$\begin{array}{l}\text { MENA } \\
(n=237)\end{array}$} & \multicolumn{2}{|l|}{$\begin{array}{l}\text { Other } \\
(n=223)\end{array}$} \\
\hline & $\overline{\mathrm{OR}}$ & $95 \% \mathrm{Cl}$ & $\overline{\mathrm{OR}}$ & $95 \% \mathrm{Cl}$ & $\overline{\mathrm{OR}}$ & $95 \% \mathrm{Cl}$ & $\overline{\mathrm{OR}}$ & $95 \% \mathrm{Cl}$ \\
\hline \multicolumn{9}{|l|}{ Step 1} \\
\hline Intention & 5.67 & $(4.10,7.85)$ & 2.49 & $(1.30,4.76)$ & 2.94 & $(2.10,4.11)$ & 2.26 & $(1.37,3.71)$ \\
\hline \multicolumn{9}{|l|}{ Step 2} \\
\hline Subjective norms & & & & & & & 3.52 & $(1.28,9.67)$ \\
\hline \multicolumn{9}{|l|}{ Step 3} \\
\hline Habit strength & & & 2.32 & $(1.13,4.78)$ & & & & \\
\hline Information processing & & & & & & & 0.43 & $(0.22,0.83)$ \\
\hline \multicolumn{9}{|l|}{ Childhood vaccination $¥$} \\
\hline Not all & 1 & & & & & & & \\
\hline All & 10.43 & $(3.05,35.69)$ & & & & & & \\
\hline Multivariable model & pseudo- $R^{2}$ & & pseudo- $R^{2}$ & & pseudo- $R^{2}$ & & pseudo- $R^{2}$ & \\
\hline Step 1 & 0.54 & & 0.20 & & 0.23 & & 0.22 & \\
\hline Step $1+2$ & & & & & & & 0.25 & \\
\hline Step $1+2+3$ & 0.56 & & 0.28 & & & & 0.29 & \\
\hline
\end{tabular}

Abbrevations: pseudo- $R^{2}$ pseudo $R$ squared, is a number that indicates how well the data fit a logistic regression model

NL denotes participants with a Dutch ethnicity, SNA denotes participants with a Surinamese, Netherlands Antillean or Aruban ethnicity, MENA denotes participants with a Middle Eastern or North African ethnicity (including Turkish participants), Other denotes participants from all other ethnicities

Interpretation of the OR of continuous determinants: If a determinant increases with one unit, we expect to see the odds to be HPV vaccinated (i.e. received 1 or 2 doses) to increase with the specified ratio for that determinant. For example, if among the Dutch intention increases with one unit, we expect to see the odds to be HPV vaccinated to increase 5.67 times

Interpretation of the OR of categorical determinants: the odds to be vaccinated are x-times higher in the non-reference categories compared to the reference category. For example, in the Dutch group if the daughter received all childhood vaccinations the odds to also become HPV vaccinated (i.e. received 1 or 2 doses) is 10.43 times higher compared to when the daughter did not receive all childhood vaccinations

${ }^{a}$ The multivariable analyses was executed by adding variables in three step: (step 1) intention, (step 2) proximal determinants, and (step 3) distal determinants. During each step backward selection was applied to reduce the number of determinants.

$¥$ Overall vaccination status for vaccinations taking place between 0 and 9 years old (HPV vaccination is offered at the age of $12-13$ years)

95\% $\mathrm{CI}=0.03,0.25]$ and higher education $[\beta=0.13 ; 95 \%$ $\mathrm{CI}=0.01,0.24]$ compared to lower education), and (9) religion ( $ß=-0.20 ; 95 \% \mathrm{CI}=-0.30,-0.10)$. In addition to the determinants found in the NL-group, we found descriptive norms $(\beta=0.13 ; 95 \% \mathrm{CI}=0.01,0.25)$ in the SNAgroup, risk perception when deciding to not vaccinate $(ß=0.11 ; 95 \% C I=0.01,0.21)$ and descriptive norms $(\beta=0.12 ; 95 \% C I=0.02,0.23)$ in the MENA-group, and descriptive norms $(\beta=0.14 ; 95 \% \mathrm{CI}=0.05,0.23)$ and having had experience with (a prestage of) cervical cancer or knowing someone close with such an experience $(B=0.20 ; 95 \% C I=0.04,0.36)$ in the Other-group. See Additional file 1: Table S4 for the selection of the variables used as input for the multivariable analyses.

The explained fraction of HPV intention $\left(R^{2}\right)$ was: 0.77 in NL-group ( 0.73 by proximal and an additional 0.04 by distal determinants), 0.66 in SNA-group ( 0.61 by proximal, an additional 0.05 by distal determinants), 0.75 in MENA-group (all by proximal determinants), and 0.66 in Other-group ( 0.63 by proximal and an additional 0.03 by distal determinants).

\section{Differences between determinants of HPV vaccination intention by ethnic group}

We tested whether the magnitude of the association of determinants of HPV vaccination intention was significantly different in one of the non-Dutch groups when compared to the NL-group (Table 5). The association of subjective norms had a stronger impact on intention in the NL-group when compared to the SNA-group $(p=0.001)$ and Other-group $(p=0.001)$; when having taken the decision regarding the HPV vaccination for an older daughter, parents/guardians from the NLgroup would more often take the same decision for the daughter now invited for vaccination, compared to parents from the SNA-group $(\mathrm{p}=0.024)$ and MENA-group $(p=0.020)$. Religion plays a significantly stronger role on $\mathrm{HPV}$ vaccination intention in the NL-group when compared to the Other-group ( $p=0.029)$.

\section{Complete case analyses}

When comparing analyses restricted to the complete cases with analyses done on the imputed dataset we 
Table 3 Odds ratios for the association between key determinants and vaccination uptake among Dutch parents/guardians, and interaction between ethnic group and these determinants. HPV vaccination acceptability study among parents/guardians, in Amsterdam, the Netherlands, 2014

\begin{tabular}{|c|c|c|c|c|c|c|c|c|}
\hline & \multirow[b]{3}{*}{$\mathrm{NL}-\mathrm{OR}$} & \multicolumn{7}{|c|}{ Interaction effect } \\
\hline & & \multicolumn{2}{|c|}{ SNA vs NI } & \multicolumn{2}{|c|}{ MENA vs NL } & \multicolumn{2}{|c|}{ Other vs NL } & \multirow{2}{*}{$\begin{array}{l}\text { Overall } \\
p \text {-value }\end{array}$} \\
\hline & & $x O R$ & $p$-value & $x O R$ & $p$-value & $x O R$ & $p$-value & \\
\hline Intention & 5.19 & 0.54 & 0.128 & 0.58 & 0.020 & 0.57 & 0.042 & 0.060 \\
\hline Subjective norms & 3.90 & 0.16 & 0.025 & 0.18 & 0.002 & 0.70 & 0.604 & 0.006 \\
\hline Habit strength & 1.36 & 1.43 & 0.415 & 0.44 & $<0.001$ & 0.58 & 0.045 & 0.001 \\
\hline Information processing & 0.83 & 0.40 & 0.141 & 1.37 & 0.265 & 0.57 & 0.153 & 0.043 \\
\hline
\end{tabular}

Childhood vaccination

Not all

All

8.52

0.33

0.320

0.65

0.647

0.30

0.159

0.519

The final multivariable model contains all variables significantly associated with HPV vaccination uptake in one of the ethnic groups

$p$-values $<0.05$ are indicated in bold

NL denotes participants with a Dutch ethnicity, SNA denotes participants with a Surinamese, Netherlands Antillean or Aruban ethnicity, MENA denotes participants with a Middle Eastern or North African ethnicity (including Turkish participants), Other denotes participants from all other ethnicities Interpretation of the OR of continuous determinants: If a determinant increases with one unit, we expect to see the odds to be HPV vaccinated to increase with the specified ratio for that determinant (i.e. received 1 or 2 doses). For example, if among the Dutch intention increases with one unit, we expect to see the odds to be HPV vaccinated to increase 5.19 times

Interpretation of the OR of categorical determinants: the odds to be vaccinated are x-times higher in the non-reference categories compared to the reference category. For example, in the Dutch group if the daughter received all childhood vaccinations the odds to also become HPV vaccinated (i.e. received 1 or 2 doses) is 8.52 times higher compared to when the daughter did not receive all childhood vaccinations. Interpretation of xOR: this is the odds ratio of the interaction factor. It indicates the factor with which the OR of the Dutch group should be multiplied to obtain the odds ratio for that determinants in that particular non-Dutch group. For example, the xOR for intention in the MENA group is 0.58 . In order to obtain the odds ratio for the MENA-group for the effect of one step increase in the intention scale on vaccination uptake, one multiplies the OR of the Dutch group (5.19) with this xOR (0.58), resulting in an OR of 3.01. So the effect of intention on vaccination uptake is significantly $(p=0.020)$ less strong than in the Dutch group

found similar results which did not affect our main conclusion (Additional file 1: Table S5 through S8).

\section{Discussion}

In all ethnic groups we found intention to be the strongest predictor of HPV vaccination uptake, explaining the largest fraction of uptake. However, the total explained variance of uptake differed across ethnic groups. In the model with intention as outcome we found attitude to be significantly associated in all groups, attitude together with all proximal determinants explained most variance of intention. We did not find direct cues for targeting different determinants in the non-Dutch groups when designing interventions aiming to improve HPV vaccination intentions and uptake. As the strength of association of the determinants with both intention and uptake appeared similar across ethnic groups, except for a number of subtleties, mostly indicating stronger associations in the NL-group when compared to the non NL-groups.

In the NL-group intention explained a much larger fraction of the variability in HPV vaccination uptake (pseudo- $\mathrm{R}^{2}=0.54$ ) when compared to the other ethnic groups (pseudo- $\mathrm{R}^{2}$ of around 0.20 ). This discrepancy can be attributed to the higher proportion of participants with a positive intention who ultimately did not go for the vaccination (inclined abstainers [26]) in the SNAgroup (11\%) and MENA-group (30\%) when compared to the NL-group (4\%) - a phenomenon that has been observed previously [27]. One might think that these discrepancies may have been caused by differential tendencies to respond in a socially desirable way to these questionnaires. However, we believe that this was not the case as we found a significantly lower intention in the MENA-group (please note this was not significantly lower in the SNA-group). An alternative explanation could be the presence of 'logistical challenges', yet the high coverage of other school-age vaccinations observed in the MENA-group and SNA-group (97\% and 95\%, respectively - see Table 1) suggest that this is not a plausible reason as these vaccinations are offered in the same way as the HPV vaccination and therefore require the same set of skills to ultimately get the vaccination. Another reason might be that parents/guardians from the MENA-group and SNA-group changed their mind in the time between completing the questionnaire and the appointment for receiving the HPV vaccination. We know that the HPV vaccination uptake in their socialcircle is generally low and therefore they have a higher chance to encounter negative beliefs, norms, and role model behavior from their peers - it is possible that parents may therefore have a higher chance to change their mind.

We did not find large differences between the strength of association of the determinants on intention across ethnic groups, except for a couple of subtleties in which 
Table 4 HPV vaccination intention: multivariable linear regression analyses of socio-demographic, social-psychological and other factors. HPV vaccination acceptability study among parents/guardians, in Amsterdam, the Netherlands, 2014

\begin{tabular}{|c|c|c|c|c|c|c|c|c|}
\hline & \multicolumn{8}{|c|}{ Multivariable $^{a}$} \\
\hline & \multicolumn{2}{|c|}{$\begin{array}{l}\mathrm{NL} \\
(n=723)\end{array}$} & \multicolumn{2}{|c|}{$\begin{array}{l}\text { SNA } \\
(n=126)\end{array}$} & \multicolumn{2}{|c|}{$\begin{array}{l}\text { MENA } \\
(n=237)\end{array}$} & \multicolumn{2}{|c|}{$\begin{array}{l}\text { Other } \\
(n=223)\end{array}$} \\
\hline & $\bar{B}$ & $95 \% \mathrm{Cl}$ & $\bar{B}$ & $95 \% \mathrm{Cl}$ & $\bar{B}$ & $95 \% \mathrm{Cl}$ & $\bar{B}$ & $95 \% \mathrm{Cl}$ \\
\hline \multicolumn{9}{|l|}{ step 1} \\
\hline Attitude & 0.66 & $(0.57,0.75)$ & 0.70 & $(0.48,0.91)$ & 1.03 & $(0.88,1.17)$ & 0.71 & $(0.55,0.86)$ \\
\hline Beliefs & 0.36 & $(0.24,0.49)$ & 0.28 & $(0.04,0.52)$ & & & 0.27 & $(0.07,0.46)$ \\
\hline Risk perception when not vaccinating & & & & & 0.11 & $(0.01,0.21)$ & & \\
\hline Relative effectiveness & 0.11 & $(0,0.22)$ & & & & & & \\
\hline Subjective norms & 0.49 & $(0.35,0.63)$ & & & 0.27 & $(0.08,0.46)$ & & \\
\hline Descriptive norms & & & 0.13 & $(0.01,0.25)$ & 0.12 & $(0.02,0.23)$ & 0.14 & $(0.05,0.23)$ \\
\hline \multicolumn{9}{|l|}{ step 2} \\
\hline Ambivalence towards the decision & -0.08 & $(-0.13,-0.03)$ & & & & & & \\
\hline Information processing & -0.10 & $(-0.15,-0.04)$ & & & & & & \\
\hline Evaluation of the HPV information & 0.23 & $(0.12,0.34)$ & 0.43 & $(0.21,0.66)$ & & & 0.25 & $(0.06,0.44)$ \\
\hline \multicolumn{9}{|c|}{ Past experience with vaccinating older daughter against HPV } \\
\hline Older daughter not & REF & & & & & & REF & \\
\hline Older daughter partially/fully vaccinated & 0.92 & $(0.65,1.19)$ & & & & & 0.94 & $(0.33,1.54)$ \\
\hline No older daughter & 0.79 & $(0.53,1.05)$ & & & & & 0.81 & $(0.21,1.40)$ \\
\hline \multicolumn{9}{|c|}{ Past experience of someone close or him/herself with (prestage of) cervical cancer } \\
\hline No & & & & & & & REF & \\
\hline Yes & & & & & & & 0.20 & $(0.04,0.36)$ \\
\hline \multicolumn{9}{|l|}{ Education } \\
\hline Low & REF & & & & & & & \\
\hline Intermediate & 0.14 & $(0.03,0.25)$ & & & & & & \\
\hline High & 0.13 & $(0.01,0.24)$ & & & & & & \\
\hline \multicolumn{9}{|l|}{ Religion } \\
\hline No religion & REF & & & & & & & \\
\hline Religious & -0.20 & $(-0.30,-0.10)$ & & & & & & \\
\hline Multivariable model & $R^{2}$ & & $R^{2}$ & & $R^{2}$ & & $R^{2}$ & \\
\hline Step 1 & 0.73 & & 0.61 & & 0.75 & & 0.63 & \\
\hline Step $1+2$ & 0.77 & & 0.66 & & & & 0.66 & \\
\hline
\end{tabular}

${ }^{a}$ The multivariable analyses was executed by adding variables in two step: (step 1) proximal determinants, and (step 2) distal determinants. During each step backward selection was applied to reduce the number of determinants. Please note, that when executing a stepwise multivariable analyses, variables significant $(p<0.05)$ in one step may not be significant when variables are added during subsequent steps

Abbrevations: $R^{2} \mathrm{R}$ squared, is a number that indicates how well the data fit a linear regression model

NL denotes participants with a Dutch ethnicity, SNA denotes participants with a Surinamese, Netherlands Antillean or Aruban ethnicity, MENA denotes participants with a Middle Eastern or North African ethnicity (including Turkish participants), Other denotes participants from all other ethnicities

Interpretation of the coefficient of continuous determinants: if a determinant increases with one unit we expect to see an increase in intention with the coefficient specified for that determinant. For example, among the Dutch, if attitude increases with one unit, we expect intention to increase with 0.66 Interpretation of the coefficient of categorical determinants: the intention to be vaccinated is $\beta$ higher or lower in the non-reference category when compared to the reference category. For example, overall, Dutch participants that are highly educated have an intention that is 0.13 higher (on the scale of -2 to +2 ) when compared to those participants with a low education

we found subjective norms and having an older daughter for which they already decided about the HPV vaccination to play a significantly stronger role in the NLgroup. We found similar proportions of explained variance and similar determinants as in a previous study on native Dutch [10]: i.e. attitude, outcome beliefs, subjective norms, ambivalence, education, religion, and past (HPV) vaccination decision making. Additionally, we found the subjective evaluation of the information provided by the national HPV vaccination campaign to be 
Table 5 Regression coefficient for the association between key determinants and vaccination intention among Dutch parents/ guardians, and interaction between ethnic group and these determinants. HPV vaccination acceptability study among parents/ guardians, in Amsterdam, the Netherlands, 2014

\begin{tabular}{|c|c|c|c|c|c|c|c|c|}
\hline & \multirow[b]{3}{*}{$N L-\beta$} & \multicolumn{7}{|c|}{ Interaction effect } \\
\hline & & \multicolumn{2}{|c|}{ SNA vs NL } & \multicolumn{2}{|c|}{ MENA vs NL } & \multicolumn{2}{|c|}{ Other vs NL } & \multirow{2}{*}{$\begin{array}{l}\text { Overall } \\
p \text {-value }\end{array}$} \\
\hline & & $\overline{\Delta \beta}$ & $\overline{p \text {-value }}$ & $\overline{\Delta B}$ & $\overline{p \text {-value }}$ & $\overline{\Delta B}$ & $\overline{p \text {-value }}$ & \\
\hline Attitude & 0.77 & -0.09 & 0.307 & 0.10 & 0.070 & -0.12 & 0.054 & 0.015 \\
\hline Beliefs & 0.36 & -0.12 & 0.268 & -0.12 & 0.173 & -0.16 & 0.051 & 0.182 \\
\hline Risk perception when not vaccinating & 0.06 & -0.10 & 0.113 & 0.08 & 0.119 & -0.08 & 0.120 & 0.020 \\
\hline Relative effectiveness & 0.12 & -0.17 & 0.192 & -0.03 & 0.735 & -0.19 & 0.050 & 0.200 \\
\hline Subjective norms & 0.44 & -0.40 & 0.001 & -0.09 & 0.335 & -0.34 & 0.001 & 0.001 \\
\hline Descriptive norms & 0.09 & -0.06 & 0.383 & 0.01 & 0.806 & -0.05 & 0.375 & 0.577 \\
\hline Ambivalence towards the decision & -0.06 & -0.01 & 0.790 & 0 & 0.975 & 0 & 0.994 & 0.995 \\
\hline Information processing & -0.11 & 0.02 & 0.717 & 0.09 & 0.081 & 0.06 & 0.261 & 0.298 \\
\hline Evaluation of the HPV information & 0.24 & 0.08 & 0.517 & -0.15 & 0.112 & -0.09 & 0.285 & 0.202 \\
\hline \multicolumn{9}{|c|}{ Past experience with vaccinating older daughter against HPV } \\
\hline Older daughter not & REF & & & & & & & 0.012 \\
\hline Older daughter partially/fully vaccinated & 0.95 & -0.65 & 0.024 & -0.54 & 0.020 & -0.27 & 0.431 & \\
\hline No older daughter & 0.81 & -0.63 & 0.017 & -0.69 & 0.001 & -0.19 & 0.561 & \\
\hline \multicolumn{9}{|c|}{ Past experience of someone close or him/herself with (prestage of) cervical cancer } \\
\hline No & REF & & & & & & & 0.093 \\
\hline Yes & 0.06 & 0.09 & 0.509 & -0.21 & 0.080 & 0.11 & 0.243 & \\
\hline \multicolumn{9}{|l|}{ Education } \\
\hline Low & REF & & & & & & & 0.282 \\
\hline Intermediate & 0.15 & 0.04 & 0.760 & -0.02 & 0.868 & -0.20 & 0.090 & \\
\hline High & 0.15 & 0.16 & 0.424 & -0.01 & 0.942 & -0.19 & 0.103 & \\
\hline \multicolumn{9}{|l|}{ Religion } \\
\hline No religion & REF & & & & & & & 0.187 \\
\hline Religious & -0.20 & 0.08 & 0.591 & 0.09 & 0.621 & 0.22 & 0.029 & \\
\hline
\end{tabular}

The final multivariable model contains all variables significantly associated with HPV vaccination intention in one of the ethnic groups $p$-values $<0.05$ are indicated in bold

NL denotes participants with a Dutch ethnicity, SNA denotes participants with a Surinamese, Netherlands Antillean or Aruban ethnicity, MENA denotes participants with a Middle Eastern or North African ethnicity (including Turkish participants), Other denotes participants from all other ethnicities

Interpretation of the coefficient of continuous determinants: if a determinant increases with one unit we expect to see an increase in intention with the coefficient specified for that determinant. For example, among the Dutch, if attitude increases with one unit, we expect intention to increase with 0.77

Interpretation of the coefficient of categorical determinants: the intention to be vaccinated is $\beta$ higher or lower in the non-reference category when compared to the reference category. For example, overall, Dutch participants that are highly educated have an intention that is 0.15 higher (on the scale of -2 to +2 ) when compared to those participants with a low education

Interpretation of $\Delta ß$ : Delta Beta is the difference in the beta in one of the non-Dutch groups when compared to the NL-group. For example, for the variable Subjective Norms, the $\Delta ß$ in the SNA-group is -0.40 , indicating that the slope is 0.40 lower in the SNA-group compared to the NL-group; this is a significant effect $(p=0.001)$

significantly associated with intention in all groups except the MENA-group.

This is the first study to present longitudinal results regarding the association of intention to vaccinate against HPV and actual (rather than self-reported) HPV vaccination in an ethnically diverse sample. Since illiteracy and language barriers are known issues in our study population [28], we anticipated this by translating the questionnaire to other languages and by offering personal assistance with completing the questionnaire. The latter might have caused some bias, yet the direction of this bias remains to be elucidated. We executed in-depth qualitative research to develop a culturally sensitive questionnaire. We made every possible effort to recruit an ethnically diverse population and enable participants to complete the questionnaire (an action that might have been beyond their comfort range). Despite these efforts our response was 33\%. Furthermore, girls whose parents/ guardians participated in this study, had a higher 
HPV vaccination uptake than the average population. However, this difference in proportions is not likely to have biased our main results as they concern associations (determinants of HPV vaccination intention and uptake) instead of point estimates of uptake or intention.

We recommend future communication strategies to target social-psychological determinants of HPV vaccination intention and uptake. Furthermore, we may conclude that intervention programs should target the same determinants for all ethnic groups, although some determinants will have stronger effects in some groups than in others. Within the scope of these determinants, the process of HPV vaccination decision making has shown to be mainly attitudinally driven, however, we should not neglect processes of internalization of social normative/media influences. As HPV vaccination intention and uptake in the different ethnic groups is based on similar determinants, future interventions could employ similar behavior change methods (e.g. psychological inoculation or peer modeling). However, the selection and design of concrete applications or strategies to reach parents in the different ethnic populations may need tailoring. For example, a prerequisite for a successful communication is that non-Dutch speaking parents/ guardians are able to understand and process the HPV vaccination information offered to them. In the MENAgroup we found that $18 \%$ completed the questionnaire in another language than Dutch and that $43 \%$ completed the questionnaire with help of someone, we therefore advise to translate this information into their native language, and to use interaction-based approaches (personal communication or social media) so parents/guardians are able to ask questions and exchange their concerns about the HPV vaccination. This also provides opportunities to correct possible misperceptions before they become salient within a particular ethnic community. Further research is needed to understand the discrepancy between intention and behavior among the non-Dutch groups. Our research stresses the need of measuring actual uptake instead of intention in an ethnical diverse population.

\section{Conclusion}

We confirmed that social-psychological factors are most decisive when parents/guardians make a decision regarding vaccinating their daughter against HPV. Based on the measured determinants we conclude that intervention programs can focus on the same determinants for all ethnic groups. Furthermore, we found the MENAgroup and SNA-group to contain a large fraction of parents/guardians that had a positive intention to vaccinate yet ultimately decided not to go for the HPV vaccination of their daughter.

\section{Additional file}

Additional file 1: Table S1. Overview of social-psychological scale measures used in the questionnaire for HPV vaccine acceptability among parents/guardians in Amsterdam, the Netherlands, 2014.

Table S2. Response rate stratified by country of birth of the mother of the invited girl, HPV vaccination acceptability study in Amsterdam, the Netherlands, 2014. Table S3. HPV vaccination uptake: bivariable logistic regression analyses of social-psychological, socio-demographic and other factors. HPV vaccination acceptability study in Amsterdam, the Netherlands, 2014. Table S4. HPV vaccination intention: bivariable linear regression analyses of social-psychological, socio-demographic and other factors, HPV vaccination acceptability study in Amsterdam, the Netherlands, 2014. Table S5. HPV vaccination uptake: multivariable logistic regression analyses of complete cases of social-psychological, socio-demographic and other factors. HPV vaccination acceptability study among parents/guardians, in Amsterdam, the Netherlands, 2014. Table S6. Complete case analyses: Odds ratios for the association between key determinants and vaccination uptake among Dutch parents/guardians, and interaction between ethnic group and these determinants. HPV vaccination acceptability study among parents/guardians, in Amsterdam, the Netherlands, 2014. Table S7. HPV vaccination intention: multivariable linear regression analyses of complete cases of socio-demographic, social-psychological and other factors. HPV vaccination acceptability study among parents/guardians, in Amsterdam, the Netherlands, 2014. Table S8. Complete case analyses: Regression coefficient for the association between key determinants and vaccination intention among Dutch parents/guardians, and interaction between ethnic group and these determinants. HPV vaccination acceptability study among parents/guardians, in Amsterdam, the Netherlands, 2014. Figure S1. Schematic representation of key dates and periods: HPV vaccination acceptability study, Amsterdam 2014 The vertical line indicates the time. Figure S2. Flow diagram of the recruitment and response of parents/guardians and their daughters of the HPV vaccination acceptability study, Amsterdam 2014. Figure S3. Proportion of girls that received two HPV vaccine doses by neighborhood in the health district of the Public Health Service of Amsterdam. (DOCX 389 kb)

\section{Abbreviations}

$\mathrm{Cl}$ : Confidence interval; HPV: Human papillomavirus; IQR: Inter quartile range; MENA: Refers to the Middle Eastern and North African ethnical group; NIP: National Immunization Program; NL: Refers to the Dutch ethnical group; OR: Odds ratio; SD: Standard deviation; SNA: Refers to the Surinamese, Netherlands Antillean and Aruban ethnical group

\section{Acknowledgments}

This HPV vaccination acceptability study was conducted by the department of child health care, the department of infectious diseases and the department of epidemiology and health promotion from the Public Health Service (GGD) of Amsterdam, in collaboration with the Expertise Center Child Health, The Netherlands Organization for Applied Scientific Research (TNO), and the department of infectious diseases epidemiology from the National Institute of Public Health and the Environment (RIVM), Bilthoven, the

Netherlands. We are most grateful to the parents/guardians who participated in this study, and the research assistants and other staff who have taken part in gathering the data for this study. We specifically thank Christa Blok (GGD), Hil Dekens (GGD), Gerben Rienk (GGD) and Petra Oomen (RIVM) for datamanagment and data collection; M. van Rooijen for designing the figures concerning the HPV vaccination uptake; M. Groentjes for the accuracy necessary for the print work; E. Vos, L. Coyer, D. Jenner, both sisters S. Aabaslama, A. Mantici and I. Oduro for enthusing parents/guardians to participate in the study and all logistical challenges and corresponding tasks necessary to invite parents/guardians. Finally, we are indebted to C. Açar, L. Boateng and S. Aabaslama who helped executing, translating, and interpreting the focusgroups which were the foundation for this questionnaire; V. van der Kamp, K. Boujbara, C. Açar, L. Boateng and S. Aabaslama and the HELIUS team for inviting parents for the focusgroups. 


\section{Funding}

This study was funded by grant 50-50405-98-121 (dossier number 204005002, Academic Collaborative) from ZonMw, the Netherlands, and a grant from the GGD Research \& Development fund of the Public Health Service of Amsterdam (project number 11-15; kp 2617).

\section{Availability of data and materials}

We welcome proposals for collaborative projects and requests for data sharing. Please note that participants' informed consent did not extend to share the data regarding actual HPV vaccination uptake. Other data can be requested via email to the corresponding author.

\section{Authors' contribution}

CJA carried out the statistical analyses and drafted the manuscript. CJA, AN, $\mathrm{YH}, \mathrm{FF}$ HM contributed to the data collection. CJA, MFSL, YH, HM, MW, AH, FF, MP and TP contributed to the study design. All authors participated in review of the manuscript, and read and approved the last version.

\section{Competing interest}

The institution of M. F. Schim van der Loeff received study funding from Sanofi Pasteur MSD; he is a co-investigator in a Merck-funded investigatorinitiated study; he is an investigator on a Sanofi Pasteur MSD sponsored trial; he served on a vaccine advisory board of GSK; his institution received in-kind contribution for an HPV study from Stichting Pathologie Onderzoek en Ontwikkeling (SPOO); his institution receives research funding from Janssen Infectious Diseases and Vaccines; other authors: no conflicts of interest.

\section{Consent for publication}

Not applicable.

\section{Ethics approval and consent to participate}

This study was approved by the Ethics Committee of the Academic Medica Centre, Amsterdam, the Netherlands (W2013_257). Participants provided informed consent by completing and returning the questionnaire. Consent to retrieve the vaccination status of the daughter was obtained by a separate question.

\section{Author details}

'Department of Infectious Diseases, Research and Prevention, Public Health Service (GGD) of Amsterdam, Nieuwe Achtergracht 100, 1018, WT, Amsterdam, The Netherlands. ${ }^{2}$ Department of Internal Medicine, Division of Infectious Diseases, Center for Infection and Immunity Amsterdam (CINIMA), Academic Medical Center (AMC), Amsterdam, The Netherlands. ${ }^{3}$ Department of Youth Health Service, Public Health Service of Amsterdam (GGD), Amsterdam, The Netherlands. ${ }^{4}$ Department of Epidemiology and Surveillance, National Institute of Public Health and the Environment (RIVM), Bilthoven, The Netherlands. ${ }^{5}$ Department of Epidemiology and Health Promotion, Public Health Service of Amsterdam (GGD), Amsterdam, The Netherlands. ${ }^{6} \mathrm{TNO}$ (Netherlands Organization for Applied Scientific Research), Expertise Centre Child Health, Leiden, The Netherlands.

Received: 29 June 2016 Accepted: 30 January 2017

Published online: 21 February 2017

\section{References}

1. van Lier EA, Oomen PJ. [Vaccination coverage of the National Immunization Programme in the Netherlands: National Institute of Public Health and the Environment (RIVM)]. 2015.

2. van Lier EA, Oomen PJ. Naccination coverage of the National Immunization Programme in the Netherlands: National Institute of Public Health and the Environment (RIVM)]. 2011.

3. Rondy $M$, van LA, Van de Kassteele J, Rust L, De MH. Determinants for HPV vaccine uptake in the Netherlands: A multilevel study. Vaccine. 2010;28:2070-5

4. Arnold M, Razum O, Coebergh JW. Cancer risk diversity in non-western migrants to Europe: An overview of the literature. Eur J Cancer. 2010;46:2647-59.

5. Downs LS, Smith JS, Scarinci I, Flowers L, Parham G. The disparity of cervical cancer in diverse populations. Gynecol Oncol. 2008;109:S22-30.

6. Howlader N, Noone AM, Krapcho M, Garshell J. SEER Cancer Statistics Review, 1975-2012. Bethesda: National Cancer Institute; 2015. http://seer. cancer.gov/csr/1975_2012/, based on November 2014 SEER data submission, posted to the SEER web site, April 2015. Accessed May 2016.

7. Fisher H, Trotter CL, Audrey S, MacDonald-Wallis K, Hickman M. Inequalities in the uptake of human papillomavirus vaccination: a systematic review and meta-analysis. Int J Epidemiol. 2013;42:896-908.

8. Marlow LA, Zimet GD, McCaffery KJ, Ostini R, Waller J. Knowledge of human papillomavirus (HPV) and HPV vaccination: an international comparison. Vaccine. 2013;31:763-9.

9. Gelman A, Miller E, Schwarz EB, Akers AY, Jeong K, Borrero S. Racial disparities in human papillomavirus vaccination: does access matter? J Adolesc Health. 2013;53:756-62.

10. van Keulen HM, Otten W, Ruiter RA, Fekkes M, Van SJ, Dusseldorp E, et al. Determinants of HPV vaccination intentions among Dutch girls and their mothers: a cross-sectional study. BMC Public Health. 2013;13:111.

11. Marlow LA, Wardle J, Waller J. Attitudes to HPV vaccination among ethnic minority mothers in the UK: an exploratory qualitative study. Hum Vaccin. 2009;5:105-10.

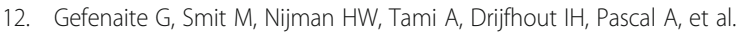
Comparatively low attendance during Human Papillomavirus catch-up vaccination among teenage girls in the Netherlands: Insights from a behavioral survey among parents. BMC Public Health. 2012;12:498.

13. Hofman R, Van EP, Richardus JH, de Kok IM, de Koning HJ, Van BM, et al. Predictors of HPV vaccination uptake: a longitudinal study among parents. Health Educ Res. 2014:29:83-96.

14. Mollers M, Lubbers K, Spoelstra SK, Weijmar-Schultz WC, Daemen T, Westra TA, et al. Equity in human papilloma virus vaccination uptake? sexual behaviour, knowledge and demographics in a cross-sectional study in (un)vaccinated girls in the Netherlands. BMC Public Health. 2014;14:288.

15. Marlow LA. HPV vaccination among ethnic minorities in the UK: knowledge, acceptability and attitudes. Br J Cancer. 2011;105:486-92.

16. Marlow LA, Wardle J, Forster AS, Waller J. Ethnic differences in human papillomavirus awareness and vaccine acceptability. J Epidemiol Community Health. 2009;63:1010-5.

17. Fishbein M, Ajzen I. Predicting and changing behavior: The reasoned action approach. New York: Psychology Press (Taylor \& Francis); 2010.

18. Bandura A. Social foundations of thought and action: A social cognitive theory. New York: Prentice Hall; 1986.

19. Becker $\mathrm{MH}$. The Health belief model and personal health behavior. Health Educ Monogr. 1974;30:324-508.

20. Stronks K, Kulu-Glasgow I, Agyemang C. The utility of 'country of birth' for the classification of ethnic groups in health research: the Dutch experience. Ethn Health. 2009;14:255-69.

21. Health statistics and information systems, Definition of region groupings. WHO.1-5-0005. http://www.who.int/about/regions/en/. Accessed May 2016.

22. Van Lier A, Oomen P, De HP, Drijfhout I, Elsinghorst B, Kemmeren J, et al. Praeventis, the immunisation register of the Netherlands: a tool to evaluate the National Immunisation Programme. Euro Surveill. 2012;17.

23. van Buuren S. Multiple imputation of discrete and continuous data by fully conditional specification. Stat Methods Med Res. 2007;16:219-42.

24. Rubin DB. Multiple Imputationfor Nonresponse in Surveys. New York: John Wile; 1987.

25. StataCorp. Stata Statistical Software: Release 14. College Station: StataCorp LP; 2015.

26. Sheeran P, Milne S, Webb TL, Gollwitzer PM. Chatper 7, Implementation Intentions and Health Behaviour. 2009.

27. Brabin L, Roberts SA, Stretch R, Baxter D, Chambers G, Kitchener $H$, et al. Uptake of first two doses of human papillomavirus vaccine by adolescent schoolgirls in Manchester: prospective cohort study. BMJ. 2008:336:1056-8.

28. Fransen M, Harris VC, Essink-Bot ML. [Low health literacy in ethnic minority patients: understandable language is the beginning of good healthcare]. Ned Tijdschr Geneeskd. 2013;157:A5581. 